\title{
语义特征图引导的青光眼篮查方法
}

\author{
郭璠 ${ }^{1)}$ ，李伟清 1 ），赵金金），邹北骥 2 ) \\ 1) (中南大学自动化学院 长沙 410083) \\ 2) (中南大学计算机学院 长沙 410083) \\ (guofancsu@163.com)
}

\begin{abstract}
摘 要: 现有的端到端青光眼笁查模型往往忽略细微病变区域而导致过拟合问题, 并且其可解释性区域尚不明确. 针对上述问题，提出一种语义特征图引导的青光眼篮查方法. 利用基于 MobileNet v2 作为特征提取网络的 DeepLab $\mathrm{v} 3+$ 分割模型进行视盘区域的分割定位，并且根据定位结果提取用于青光眼䇻查的重点感兴趣区域，再通过设计注意 力模块将其嵌人 VGG 分类网络实现青光眼的准确篮查. 注意力模块利用语义特征图融合浅层特征综合生成注意力 图，通过强化特征分类相关区域而抑制非相关区域，达到增强模型分类鲁棒性的目的. 所提方法基于 LAG database 数据集测试的敏感性、特异性以及 AUC 分别为 $0.970,0.983$ 和 0.996 , 优于已有方法, 且通过可视化注意力激活热图 得到的模型决策关注区域更为精细，能够获得与医生诊断参考区域相一致的结果.
\end{abstract}

关键词: 青光眼笁查; 可解释性; 注意力机制; 卷积神经网络; 决策关注区域

中图法分类号: TP391.4 DOI: 10.3724/SP.J.1089.2021.18474

\section{Glaucoma Screening Method Based on Semantic Feature Map Guidance}

\author{
Guo Fan ${ }^{1)}$, Li Weiqing ${ }^{1)}$, Zhao Xin ${ }^{2)}$, and Zou Beiji ${ }^{2)}$ \\ 1) (School of Automation, Central South University, Changsha 410083) \\ ${ }^{2)}$ (School of Computer, Central South University, Changsha 410083)
}

\begin{abstract}
Existing end-to-end glaucoma screening models don't pay enough attention to the region with subtle lesions, and this leads to over-fitting. Besides, the interpretability of the existing models is not clear. Therefore, a glaucoma screening method based on semantic feature map guidance is proposed in this paper. We first adopted the DeepLab v3+ segmentation model using MobileNet v2 as the feature extraction network to locate the optic disc. Then we extracted the region of interest for glaucoma screening based on the positioning result. Finally, an attention module was designed and embedded in the VGG classification network to achieve accurate glaucoma screening. The attention block of the method uses the semantic discrimination of the semantic feature map and the shallow feature fusion to generate the attention map. Thus, the areas that related to the shallow feature map classification can be strengthened and the non-related areas will be suppressed to enhance the robustness of the model classification ability. The sensitivity, specificity and AUC of the proposed method tested on the LAG database data set are $0.970,0.983$ and 0.996 , respectively, indicating that the screening accuracy of the proposed method is relatively higher than that of the existing methods. Besides, the model decision attention areas obtained by visualizing the attention activation map are more accurate and consistent with the doctors' diagnosis reference regions.
\end{abstract}

收稿日期：2020-07-04; 修回日期：2020-12-12. 基金项目：国家自然科学基金青年科学基金(61502537); 湖南省自然科学基金 (2018JJ3681); 中南大学研究生自由探索与创新项目(2020zzts567). 郭璠(1982-), 女, 博士, 副教授, 硕士生导师, CCF 会员, 主要研 究方向为图像处理、计算机视觉、人工智能; 李伟清(1997一), 男, 硕士研究生, 主要研究方向为医学图像处理; 赵金金(1994-), 男, 硕 士, 主要研究方向为医学图像处理; 刍北䩀(1961一), 男, 博士, 教授, 博士生导师, CCF 会员, 主要研究方向为图像处理、计算机视觉. 
Key words: glaucoma screening; interpretability; attention scheme; convolutional neural networks; decision attention area

青光眼疾病已经发展成为世界第二大不可逆 的致盲眼科慢性疾病 ${ }^{[1]}$, 大多患者发病过程并未有 任何不适，而发现时已发展为晚期，故该病也被称 为视觉的无声小偷 ${ }^{[2]}$. 尽管无法治愈, 但早发现、 早治疗可有效地降低致盲率，因此早期篎查成为 应对该疾病的重要方法。

在临床上，眼内压检测、视野测量以及视乳头 检查成为评估青光眼程度的普遍方法. 而部分人 群天生眼内压高，导致眼内压检测笁查并不严谨. 视野测量同样存在一定测量误差, 这使得视乳头 检查成为主流篎查方法 ${ }^{[3-4]}$. 数字眼底照相机所拍 摄的眼底图像由于成像质量较高且价格便宜成为 眼科医师篮查设备首选. 同时, 篮查青光眼是一个 耗时的过程，依赖专业眼科医生完成大规模青光 眼篎查，费时、费力且篮查质量难以保障. 因此, 计算机辅助箴查完成青光眼早期检测至关重要. 已有篮查方法可依据篮查方式分为基于临床指标 的篮查方法和基于图像识别的筛查方法 2 大类.

青光眼发展过程中往往伴有视杯扩张趋势, 在临床中杯盘比与青光眼严重程度呈正相关关系. 因此基于临床指标的篮查方法侧重于与青光眼发 展程度密切相关的临床指标量化分析, 包括杯盘 比 $^{[5]}$ 、下上鼻颢 (inferior superior nasal temporal, ISNT)准则和盘沿分布相关指标等. 视盘视杯主要 是通过如形态学变换的图像处理方法、像素点聚类 等传统方法, 或者基于深度学习卷积神经网络模 型来完成分割. Walter 等 ${ }^{[6]}$ 将图像由 RGB 颜色空间 转到 HLS 颜色空间, 基于亮度通道采用阈值技术 定位视盘, 最后在眼底图像 RGB 颜色空间的 R 通 道通过分水岭分割算法确定视盘边界. Nayak 等 ${ }^{[7]}$ 先在眼底图像的 RGB 颜色空间的 R 通道通过形态 学处理分割出视盘, 再在 $\mathrm{G}$ 通道以同样的方式分 割出视杯, 最后计算坚直方向杯盘比, 以评估青光 眼严重程度. $\mathrm{Xu}$ 等 ${ }^{[8]}$ 根据平滑度、梯度方向和灰度 中值信息重新定义轮廓关键点的矢量表达, 以一 种改进的轮廓能量函数来提取视盘. $\mathrm{Li}$ 等 ${ }^{[9]}$ 和 $\mathrm{Kim}^{[10]}$ 等也采用类似的主动轮廓方法对视盘边界 建模, 但视盘旁的生理病变会干扰主动轮廓模型, 从而导致视盘边界定位不准.

Cheng 等 ${ }^{[11]}$ 提出一种基于超像素分类的视盘 视杯分割算法，首先使用简单线性迭代聚类算法 聚集周边像素生成超像素. 在视盘分割任务中，用 基于支持向量机(support vector machine, SVM)的 机器学习模型实现超像素分类. 视盘超像素判别 模型从直方图和中心周边统计 2 个方面提取相关 特征. Zheng 等 ${ }^{[12]}$ 提出一种通用能量函数, 结合图 割算法实现视盘视杯边界的提取. 不同于以往工 作，该模型同时将视盘和视杯的位置、形状、轮廓 厚度, 以及视盘包含视杯这些先验信息融人其中 相互作用, 起到优化视盘和视杯分割效果的作用. 在文献[13-15]中都使用类似的U型深度卷积网络对 视盘和视杯结构进行分割, 但是, $\mathrm{Fu}$ 等 ${ }^{[13]}$ 在 $\mathrm{U}$ 型网 络的编码层同时将眼底图像的多尺度信息融合起 来提取特征, 并在解码层采用深层监督的方式提 升视盘视杯分割准确率. 实验结果表明, 通过极坐 标转换将眼底图像转换到极坐标系可进一步提升 分割精度. 此外, $\mathrm{Xu}$ 等 ${ }^{[16]}$ 使用变分自编码或者视 杯重构的方式确定视盘视杯边界.

基于视盘视杯形状和亮度分布特征并通过传 统聚类与分割算法能实现病灶分割, 但其易受到 血管分布、伪影以及对比度影响, 造成边界定位不 准. 此外, 分割误差对最终青光眼篮查造成不可评 估的影响. 本文采用端到端的方法检测青光眼, 可 有效地避免由于分割误差所造成的篎查误诊.

直接基于图像识别的篮查方法针对特征提取 方式可分为基于手工特征和基于深度学习的篮查方 法. 基于手工特征的篮查方法依赖病变相关特征的 量化表征, Agarwal 等 ${ }^{[17]}$ 先通过自适应阈值技术在 RGB 颜色空间 $\mathrm{G}$ 通道提取视盘和视杯区域, 并依 据视盘视杯分割掩模计算出杯盘比、盘沿视盘面积 比和盘沿视盘厚度比值作为临床指导特征, 训练 SVM 模型作为青光眼分类模型. 此外, 基于 Gabor 变换和离散小波变换提取的能量特征 ${ }^{[18-20]}$, 基于高 阶累积特征 ${ }^{[21]}$ 的自动青光眼篮查模型被提出, 这 些工作都是从图像隐性特征角度挖掘青光眼特征. 这类方法一般会先定位视盘，并将包含视盘的局 部区域截取出来作为研究对象, 通过 Gabor变换或 离散小波变换提取纹理性特征来进行分类建模, 其中均值方差和能量信息一般用于表征变换后的 特征图像. 这样的做法显然会丢失大量细节信息 仅保留全局纹理信息，单纯依赖这些特征篮查青 光眼往往鲁棒性不强. 利用高阶光谱与离散小波 变换特征用于青光眼篮查 ${ }^{[22]}$, 其篮查模型为基于 多项式核的 SVM 模型, 并且该模型在私有数据上 
得出 $95 \%$ 的准确率.

将视盘感知的融合网络 (disc-aware ensemble networks, DENet)模型 ${ }^{[23]}$ 用于青光眼篮查，它通过 4 条检测分支分别从全局眼底图、视盘感兴区域、 极坐标变换下的视盘区域以及分割引导特征独立 地进行青光眼篮查，最终的篮查结果由这 4 条预测 分支投票决定. 在公开数据集实验结果显示，融合 模型得出篮查准确率有显著提升. 基于数字眼底 图像数据和眼底病例报告数据, Chai 等 ${ }^{[24]}$ 提出一 种基于图像隐性特征与领域知识相结合的方法, 实现智能青光眼節查多分支神经网络(multi-branch neural networks, MB-NN). 该方法同 DENet ${ }^{[23]}$ 相 似，都是从眼底图全图角度和视盘局部区域提取 特征并进行特征融合; 不同的是该方法还从眼底 报告中提取年龄、眼内压、是否出血、是否视野模 糊等 28 项临床诊断指标作为辅助特征辅助分类.

基于深度学习的篎查模型采用数据驱动方式 自动完成特征工程与分类建模，在数据充足情况 下分类性能往往优于传统手工特征建模, 但已有 卷积分类模型仍有一些不足之处. 首先, 已有模型 多采用迁移学习微调分类模型，而未充分关注对 分类诊断影响较大的细微病变相关区域对分类模 型的影响，导致模型易过拟合. 其次，现有基于深 度学习篮查方法决策解释性证据不明显, 这使得 医生无法完成对模型完成决策可靠性检验.

本文针对已有方法的不足，提出一种端到端 的语义特征图引导的青光眼篮查方法, 避免了篮 查方法繁杂所造成的误差累积. 具体而言, 该方法 首先采用基于 DeepLab v3 ${ }^{[25]}$ 的语义分割模型实
现视盘定位, 以 MobileNet ${ }^{[26]}$ 为主干模块使得分割 模型轻量且高效. 基于定位结果裁剪感兴趣区域, 并提出语义特征图引导的青光眼分类模型，通过 注意力机制融合多尺度特征, 以强化细微病变相 关区域特征而抑制非相关区域，从而提升模型预 测准确率; 通过注意力激活热图可视化来为模型 决策提供证据图谱, 可为医生提供模型决策过程 的可靠性评估.

\section{1 本文方法}

\section{1 方法框架}

本文方法包含视盘定位与青光眼分类 2 部分, 通过分割视盘区域间接定位, 并基于分割结果裁 剪出用于青光眼篎查的感兴趣区域.

该分割方法为一个基于 MobileNet $\mathrm{v} 2^{[26]}$ 作为特 征提取网络的 DeepLab v3+分割模型 ${ }^{[25]}$. MobileNet v2 网络结合深度分离卷积与倒置残差结构使得模 型轻量化. 分割模型利用多孔金字塔池化模块 (atrous spatial pyramid pooling, ASPP)充分挖掘多 感受野特征, 以实现精准分割.

青光眼分类模型的整体结构与经典深度卷积 分类模型相类似, 以 VGG 模型 ${ }^{[27]}$ 作为研究对象, 巧妙地将深度语义特征图的空间性与语义性作为 浅层特征引导模式生成注意力区域, 以注意力图 强化分类感兴趣区域而抑制非相关区域，以突出 浅层局部细节特征，最终融合多尺度特征来完成 分类建模. 本文方法流程图如图 1 所示.

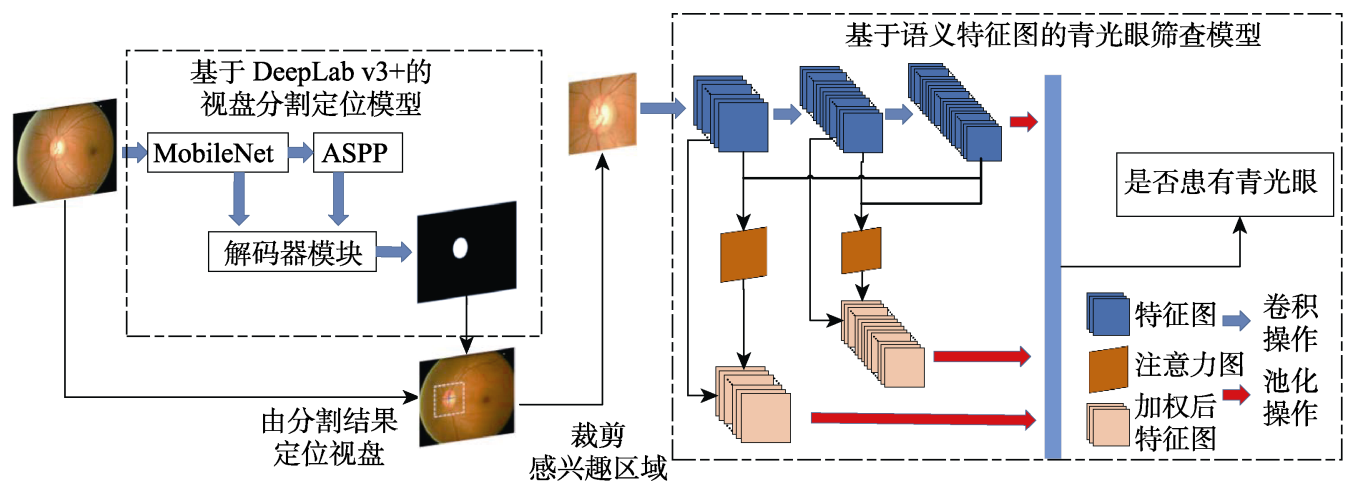

图 1 本文方法流程

\section{2 轻量级视盘定位网络}

考虑与青光眼相关的判别性区域位于视盘周 边，有效地提取该感兴趣区域并剔除非相关区域, 对最终分类模型的计算效率以及准确度有着重要 的影响. 传统的定位方法主要采用形态学变换提
取血管与高亮区域，最后以滑动窗口技术实现视 盘定位. 这样的定位方法完全依赖手工特征的提 取, 大部分情况下其在定位准确度和性能上较优 异, 但对于较为模糊、血管分布不清晰、对比度较 差的眼底图或者视盘旁病变严重的眼底图, 传统 
的定位方法则难以适应.

为了应对以上挑战，本文提出采用基于深度 卷积的视盘分割模型进行高效视盘定位. DeepLab $\mathrm{v} 3+$ 模型为一种基于全卷积操作的语义分割模型, 模型结构图如图 2 所示. 其中, Conv 表示卷积操作, $R$ 为空洞率. 该模型将常见分类模型的特征提取 部分(主干模块)作为自己的分割特征提取模块，可 作为主干模块的有 $\operatorname{ResNet}^{[28]}$, DenseNet ${ }^{[29]}$ 以及 Xception ${ }^{[30]}$ 等经典分类模型.

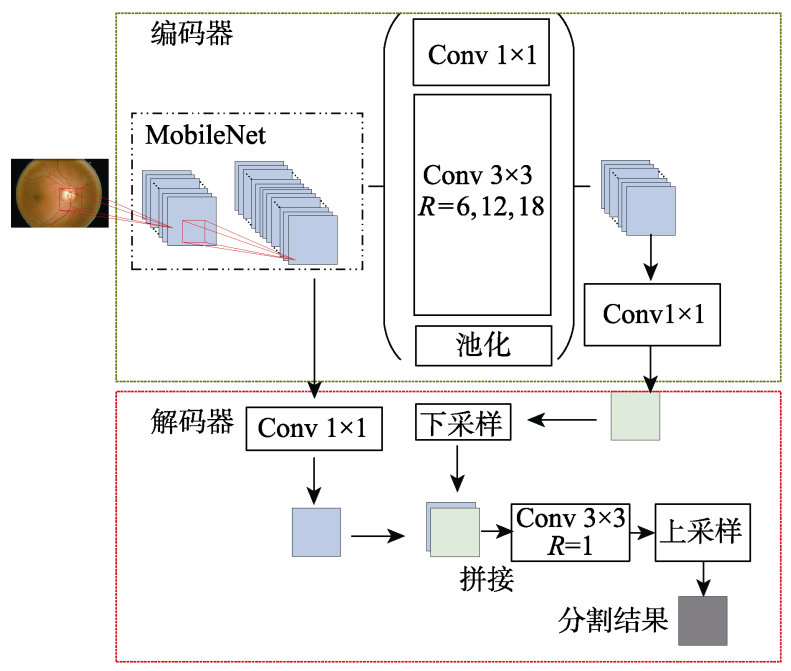

图 2 基于 DeepLab v3+的视盘分割定位模型

在分割任务中预测输出为与输人原图等大小 的分割掩模, 因此在语义分割模型中往往以 $\mathrm{FCN}^{[31]}$ 类似结构的全卷积模型设计, 这样的设计 使得模型可适应任意尺度的输人图像. 在 DeepLab $\mathrm{v} 3+$ 中，在特征提取模块后为 ASPP 模块.

ASPP 模块采用一组不同空洞率的空洞卷积核 提取给定输人特征图不同尺度的语义特征, 并对 给定输人特征图分别进行全局平均池化、 $1 \times 1$ 卷积 以及双线性上采样后，获取特征图; 将上述步骤获 取的所有特征图进行拼接, 再经过 $1 \times 1$ 卷积进行通 道调整，得到整个模块的输出特征图. 每一步卷积 操作后都进行了 ReLU 激活和批归一化处理(batch normalization, BN), 其结构如图 3 所示.

空洞卷积的数学定义为

$$
y[i]=\sum_{k} x[i+R k] w[k]
$$

其中， $x$ 表示输人特征图; $y$ 表示卷积操作的输 出; $w$ 表示卷积核; 空洞率 $R$ 表示对输人信号进 行采样的步幅. 标准卷积是空洞率 $R=1$ 时的特例. 图 4 展示了具有不同空洞率的空洞卷积示意图.

为了提高边缘区域分割准确率，在 DeepLab

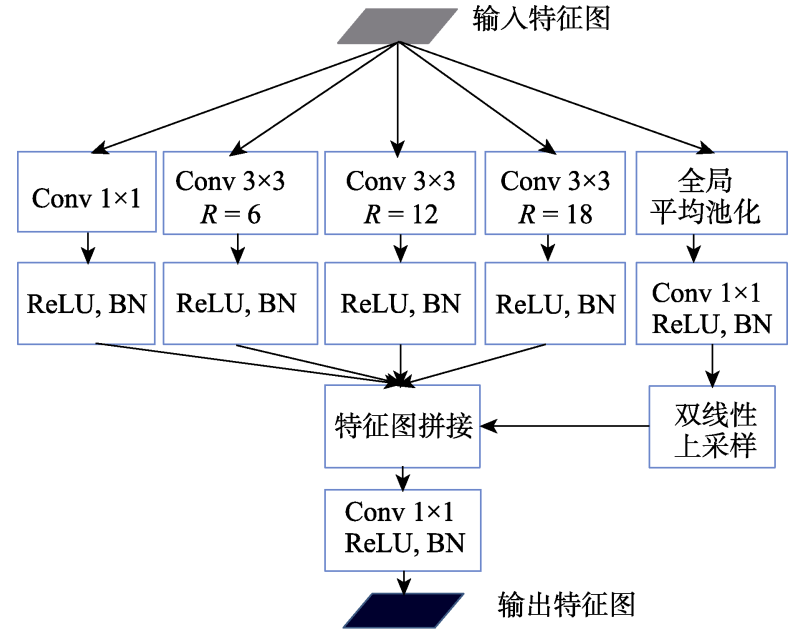

图 3 ASPP 模块结构图
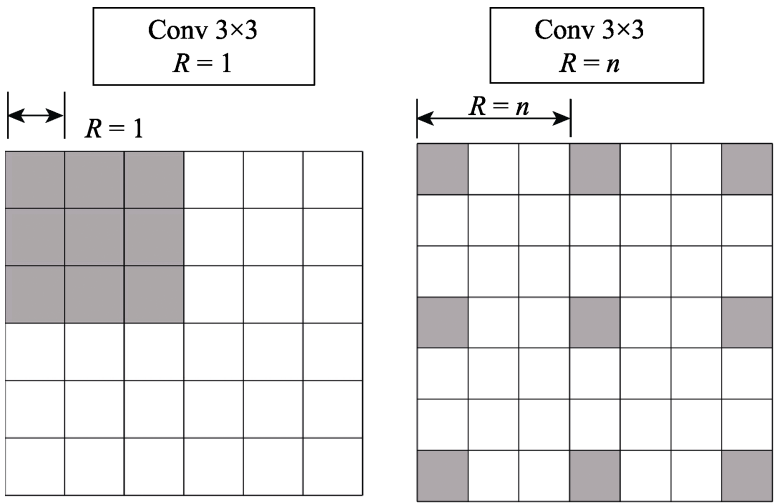

图 4 具有不同空洞率的空洞卷积

$v 3+$ 中将 ASPP 模块所得特征表征看做编码之后的 高阶特征图, 并将特征图与特征提取主干网络较 为浅层的特征图融合. 例如, 将 ResNet 中第 2 个残 差提取模块的输出特征图作为浅层特征表征后, 融合高阶语义特征与低阶浅层特征, 进一步提高 分割精度. 由于低阶浅层特征语义性较差、空间性 较强, 而高阶语义特征对最后的分割输出更为重 要; 因此在特征融合前先对浅层特征通过 $1 \times 1$ 卷积 来降维，再通过拼接的方式组合特征; 最后进行 $3 \times 3$ 的卷积操作, 输出预测分割结果, 如图 2 中解 码器部分所示.

通过空洞卷积可以在不缩减特征图尺度下增大 感受野，但是变大的特征图对后续的计算带来成倍 的开销. 考虑视盘分割任务较为单一且本分割模型 最终任务为定位, 选用轻量级 MobileNet v2 模型[26] 作为特征提取主干网络. MobileNet v1 使用深度分离 卷积与 $1 \times 1$ 卷积替换标准卷积操作, 可成倍减少计 算量. 深度可分离卷积是指输人特征图的每个通道 都对应一个卷积核, 这样输出的特征的每个通道只 与输人特征图对应的通道相关, 其结构如图 5 所示. 


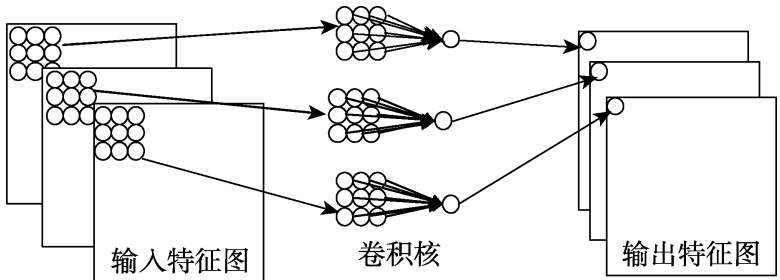

图 5 深度分离卷积示意图

在深度分离卷积的基础上, MobileNet v2 提出 倒置残差模块以及线性瓶颈设计, 在进一步减少 计算量的同时，有效地提升模型精度. MobileNet $\mathrm{v} 2$ 的基础计算模块如图 6 所示，先通过 $1 \times 1$ 的卷积 操作对输人特征图放大特征维度并激活, 从而丰 富特征表征; 接着进行深度分离卷积与特征激活 来提取单通道特征; 最后通过 $1 \times 1$ 卷积进行线性降 维, 注意到所有激活函数均采用 ReLU6 激活函数. 此外, 在倒置残差卷积模块的输出端并不使用激 活函数。实验表明，激活输出易造成卷积核失活， 从而降低最终精度.

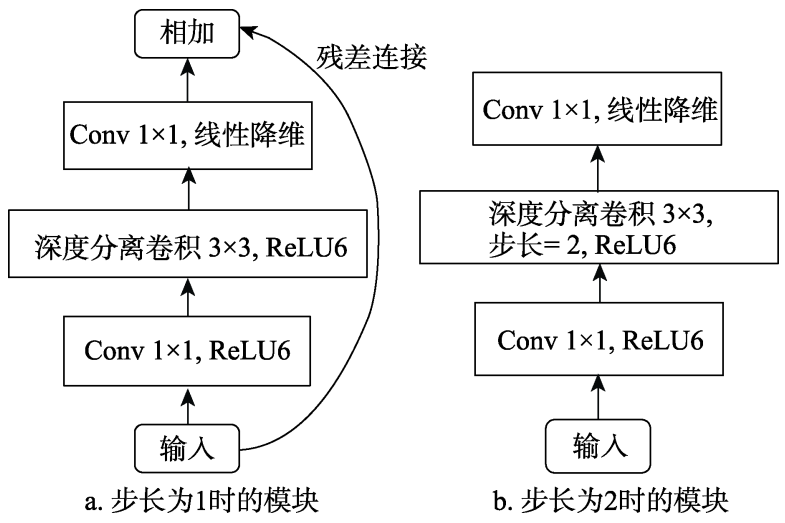

图 6 MobileNet v2 的基础计算模块

实验中，输出层采用 Sigmoid 函数激活，用于训 练 DeepLab v3+模型的损失函数为交叉熵损失, 即

$$
L_{\mathrm{CE}}=-\frac{1}{N} \sum_{i=1}^{N}\left[y^{i} \cdot \lg p^{i}+\left(1-y^{i}\right) \cdot \lg \left(1-p^{i}\right)\right]
$$

其中, $p^{i}$ 表示图像样本 $i$ 预测为正样本的概率; $y^{i}$ 表示像素 $i$ 的真实标签值.

图像随机翻转、随机旋转、随机对比度和随机 裁剪，这些数据增强手段在训练阶段增强样本多 样性，抑制模型过拟合. 将视盘定位问题转换成视 盘分割问题，采用 DeepLab v3+语义分割模型结 构, 配合 MobileNet v2 作为特征提取主干模块, 使 得模型轻量化. 实验表明，基于深度全卷积网络的 视盘分割方法对视盘定位鲁棒性有显著的提升. 通过视盘定位的结果，从全局眼底图像中裁剪约 为最大视盘区域 2 倍的方形区域，作为后续青光眼
分类检测工作的感兴趣区域，如图 7 所示.

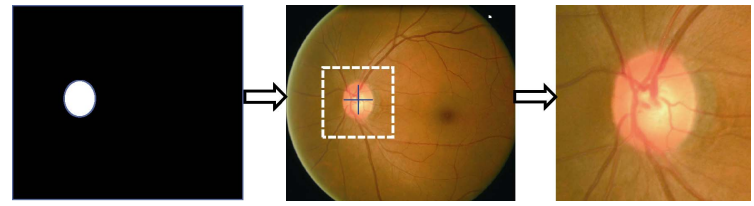

图 7 获取青光眼分类检测感兴趣区域

\section{3 语义特征图引导的青光眼分类模型}

随着近些年深度学习的飞速发展，卷积神经 网络模型在图像分类、检测及分割等任务大放异 彩，越来越多的医学影像分析任务也开始尝试基 于深度学习模型突破已有方法的局限. 在自然图 像分析中，经典的 $\mathrm{VGG}^{[27]}$, ResNet ${ }^{[28]}$, DenseNet ${ }^{[29]}$ 等模型已在 ImageNet 数据集上接近或突破人类识 别的准确度. 经典卷积深度模型或者以它们的基 础模块所设计的卷积网络模型也逐渐被应用于青 光眼篎查研究. 在青光眼篎查方向应用最多的是 经典分类网络与迁移学习的结合. 在医学影像中, 由于医学影像标注的严谨性与专业性导致数据的 稀缺, 规模一般为几十乃至几百幅. 相比于 ImageNet 数百万数据规模, 青光眼所能用于训练 深度学习模型的数据过于稀少, 导致模型趋向严 重的过拟合. 而迁移学习, 也叫微调, 是深度学习 中最简单的网络迁移方法. 利用 ImageNet 大规模 数据训练出来的模型权重作为青光眼分类任务的 初始权重，能有效地加速模型收玫并提升准确率.

在眼底图像中，青光眼相关的病变区域多为 非常细微的局部区域. 例如, 视杯扩散现象、盘沿 分布变化或者视盘出血和视神经纤维层损伤等, 这些局部病变区域非常容易在深度卷积模型的多 次池化操作中丢失，使得有效的判别性局部区域 被部分忽略, 导致最终的分类性能难以进一步提 升，模型趋向过拟合. 此外，基于深度学习模型的 可解释性在医学影像分析中也格外受关注, 如何 让模型在保证篎查精度的同时给出解释性证据, 成为青光眼篮查的另一难点.

基于上述问题，本文提出一种语义特征图引 导的青光眼笁查模型，通过注意力机制将深度卷 积模型的深层语义性及空间性强的特征图作为引 导特征, 对较为浅层特征进一步优化; 通过浅层特 征图与深层特征图自我学习出的注意力图, 对浅 层特征中局部分类相关区域进行强化，并抑制非 相关区域，以保留模型的局部细节特征来辅助判 别性特征提取, 提高最终的分类性能. 本文的语义 特征图引导的青光眼分类模型结构如图 8 所示, 模 型的输人为感兴趣区域, 输出为青光眼患病概率. 


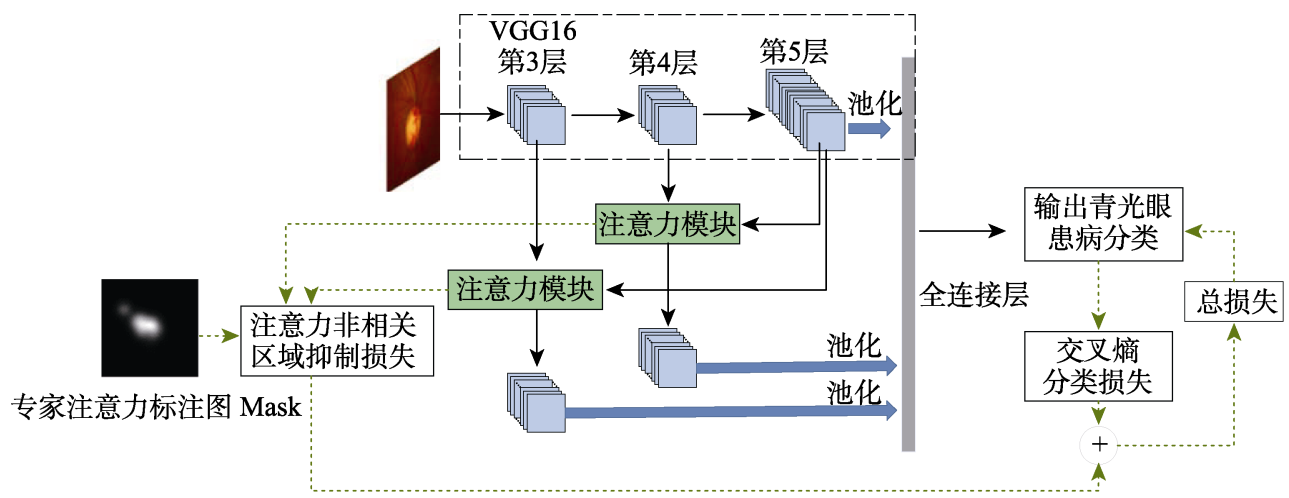

图 8 语义特征图引导的青光眼篎查模型

\subsection{1 语义特征引导的注意力机制}

常见的卷积神经网络分类模型呈现为序列状, 模型一般由多个基础计算模块构成。例如，在 ResNet 中残差模块为主要模块, DenseNet 主要由 密集连接模块以及转换模块组成. 这些基础计算 模块主要由卷积、 $\mathrm{BN}$ 层、激活函数以及池化操作 构成. 池化操作每隔一段卷积操作后被添加用于 减少特征图尺度，这样的操作也会带来额外的平 移不变性. 深度学习模型也是一种层次表示学习 算法, 可以将卷积深度学习模型不同深度特征图 看成不同语义层次的表征. 其中, 浅层特征图尺度 偏大，用于边缘等基础特征提取; 而深层特征图尺 度偏小，但用于表示抽象的语义概念，且保持一定 空间位置相对关系.

眼底图像中与青光眼相关的病变区域非常细 微, 类似于盘沿变化、视盘出血、视神经纤维层 损伤等这些病变区域极易在多次池化操作后丢 失。考虑模型高阶特征的语义性及空间性特点, 本文提出一种注意力机制用于强化浅层特征图中 分类强相关区域而抑制非相关区域，以弥补高阶 语义特征细节特征的丢失，其注意力模块示意图 如图 9 所示. 该模块的输人为深层和浅层 2 组特
征图, 首先通过卷积操作将 2 组不同特征维度的 特征图映射到同一维度特征空间，并通过双线性 插值的方法统一特征图尺度; 采用逐像素相加的 方式融合 2 组特征图, 并通过卷积操作生成浅层 特征图对应的单通道注意力图. 将深层语义特征 图和浅层语义特征图结合构建注意力模块, 可以 兼顾空间信息和纹理信息，有助于注意力图的生 成. 注意力图采用 Softmax 操作激活, 最终将注 意力图与浅层特征图逐像素相乘, 得到注意力模 块的输出特征图. 因为浅层特征图主要包括目标 边缘特征和空间信息, 采用注意力图对浅层特征 图进行加权可以强化青光眼篮查的相关区域而抑 制非相关区域，使得关键特征可以在浅层特征图 中得到加强.

在训练过程中, 为了引导注意力模块的自我 学习, 采用专业眼科医生标注的局部感兴区域即 注意力掩模图作为注意力模块中的目标输出图, 并且提出一种注意力非相关区域抑制损失函数, 计算注意力模块的输出和目标注意力掩模图的损 失值, 将其作为模型总损失的一部分. 该损失使得 模型在训练过程中可以聚焦于注意力模块参数的 更新, 更好地构建注意力模块, 以提升网络对于青

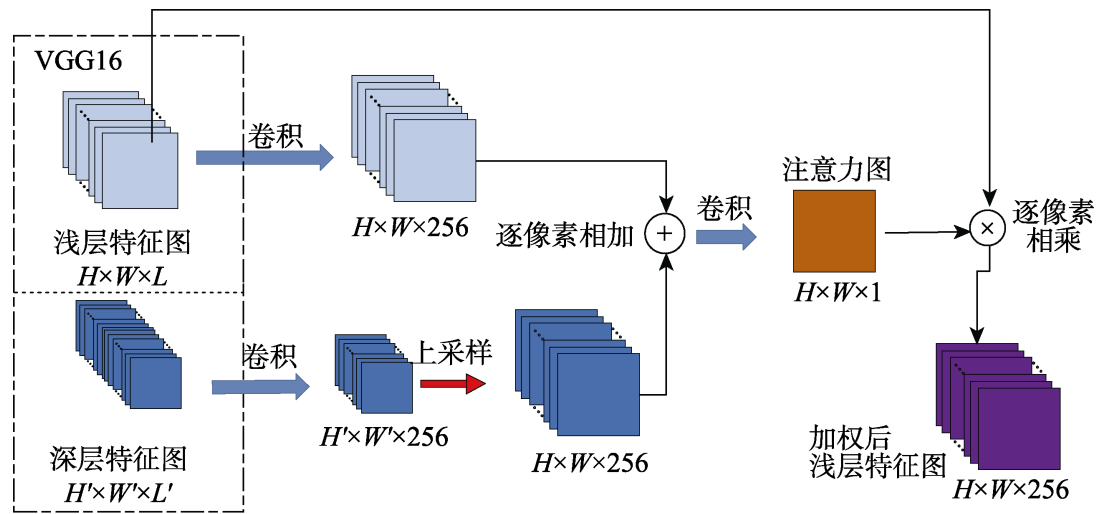

图 9 语义特征图引导注意力模块示意图 
光眼关键患病特征的提取效果.

本文方法以 VGG16 为基础模型. VGG16 模型 基础模块为堆叠的 $3 \times 3$ 大小的卷积操作, 每隔一 段卷积操作插人池化操作来降低特征图尺度. 依 据特征图大小, 将 VGG16 模型同样大小的特征图 分在同一组, 这样 VGG16 模型分成 5 组. 其中, 第 5 组特征图为语义性最强但尺度最小的特征图, 因 此将这组特征图当作引导特征图. 考虑想要强化 的特征图需要兼顾一定的语义性与尺度大小，第 3 组及第 4 组特征图成为较好的选择，因此本文分别 针对第 3 组及第 4 组特征构造 2 组注意力模块. 第 1 组注意力模块将第 3 组特征作为浅层特征, 第 5 组特征作为语义特征构建注意力图, 而第 2 组注意 力模块将第 4 组特征与第 5 组特征结合构建注意力 图, 2 组注意力图分别对对应的浅层特征图加权优 化, 如图 8 所示.

通过注意力模块整合 VGG16 模型中第 5 组及 第 3 和第 4 组特征图, 能够得到全局语义与局部显 著特征图. 不同于经典卷积分类网络只使用最后 的语义特征图作为分类特征，本文方法将第 3 和第 4 组被强化后的特征图与第 5 组的全局语义特征图 融合起来, 作为分类特征进行建模. 具体方式为对 这 3 组特征图分别进行全局均值池化, 然后拼接所 得特征向量, 再连接 dropout 层、全连接层进行青 光眼与非青光眼分类. 其中, dropout 层为深度学习 中常见的抑制过拟合操作，而最后输出层采用 Softmax 操作获得预测概率.

综上所述，融合深层语义特征图与浅层特征 图以生成注意力图, 可起到强化局部分类相关特 征而抑制非相关特征效果，这样的设计正是为了 突出眼底图像中局部细节区域对分类的作用. 此 外, 训练好的青光眼篮查模型通过可视化注意力 图展示模型关注区域, 可起到较好的模型自我解 释作用.

\subsection{2 分类及注意力图损失函数设计}

训练分类模型涉及分类损失与注意力图损失 2 个损失函数. 青光眼分类所用的损失函数为交叉 熵损失函数, 其计算公式如式(2)所示. 本文注意 力非相关区域抑制的损失函数为

$$
L_{\text {attention }}=-\frac{1}{N} \sum_{i=1}^{N}\left(1-M_{\text {target }}^{i}\right) \cdot \lg \left(M_{\text {pred }}^{i}\right)
$$

其中, $M_{\text {target }}$ 为依据专家给出的局部感兴趣区域或 者依据先验信息得出的注意力二值图; $M_{\text {target }}^{i}$ 表示
注意力图的第 $i$ 个像素值, 若为感兴趣区域, 则 $M_{\text {target }}^{i}=1$; 否则, $M_{\text {target }}^{i}=0$. 通过 1- $M_{\text {target }}^{i}$ 求出 所有非感兴趣区域, 将模型预测结果 $M_{\mathrm{pred}}^{i}$ 取对数作 为预测损失. 最后本文方法只关注非感兴趣区域的 激活，通过式(3)的激活损失抑制预测注意力图上非 相关区域的激活，而不干扰感兴趣区域的激活模式, 该损失引导模型对于病理区域的预测朝着先验区域 靠近.

模型总损失为

$$
L=\lambda_{1} L_{\mathrm{CE}}+\lambda_{2} L_{\text {attention }}
$$

其中， $L_{\mathrm{CE}}$ 为交叉摘损失; $L_{\text {attention }}$ 为注意力非相关 区域抑制损失; $\lambda_{1}$ 和 $\lambda_{2}$ 表示 2 种损失的权重, 本文 中均被设置为 0.5 .

\section{2 实验结果与分析}

为全面评估青光眼篮查方法的有效性，实验 采用 3 个包含青光眼专家标签的公开眼底数据集 LAG database ${ }^{[32]}$, REFUGE ${ }^{[33]}$ 和 DRISHTI-GS1 ${ }^{[34]}$, 同时采用敏感性、特异性以及 AUC 指标来评估各 种方法. 此外, 本文还基于 LAG database 数据集进 行了构造注意力机制模块的比较实验，以及视盘 分割定位模块的消融实验.

\section{1 实验数据集}

LAG database 是近期被公开的最大的包含青 光眼专家标签的眼底图像数据集, 该数据集已公 开 4854 幅彩色眼底图像，有 1711 幅疑似青光眼 患者眼底图像和 3143 幅非青光眼眼底图像. 该数 据集中所有眼底数据均由合格的青光眼专家综合 考虑眼内压、视野以及视盘病变而得出诊断结果. 此外, 该数据集还通过捕获专家诊断过程中专家 注意力区域并以此生成注意力图. 该注意力图为 青光眼䇻查方法提供了良好的先验信息, 数据集 中眼底图像及对应注意力区域如图 10 所示.

REFUGE 数据集为 REFUGE 竞赛所提供的眼 底图像数据集, 它包含 1200 幅彩色眼底图像, 其 中训练、验证和测试数据分别有 400 幅. 数据均由 2 种不同型号眼底照相机生成, 它们在亮度、对比 度以及尺度均有较大差距. 训练集与验证集都分 别含有 40 幅青光眼和 360 幅非青光眼图像，而测 试集未提供标签数据. DRISHTI-GS1 数据集包含 101 幅眼底图像, 其中 70 幅为青光眼眼底图像, 31 幅为正常眼底图像。 


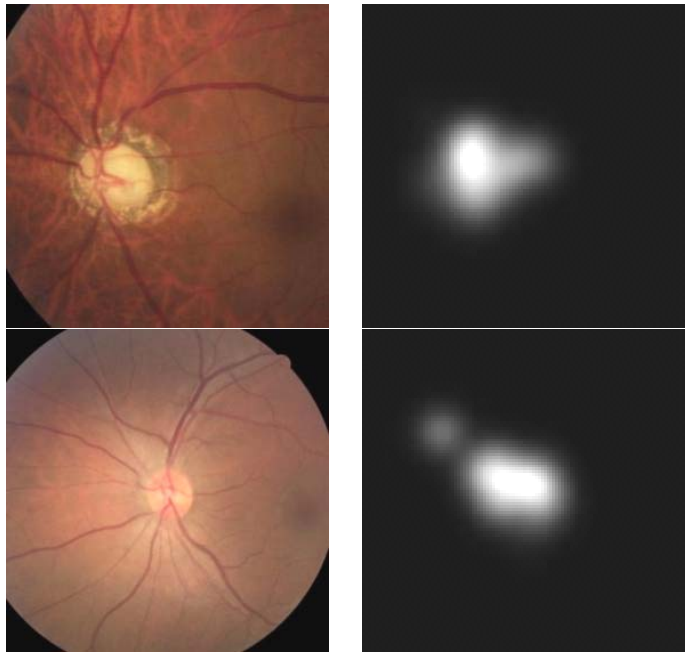

图 10 LAG database 数据集中眼底图像与对应的 专家诊断注意力图

考虑 REFUGE 与 DRISHTI-GS1 数据集数据规 模偏小, 而 LAG database 规模偏大, 训练深度学习 模型所需数据偏多, 本文将 LAG database 数据集 依据 8:2 比例随机划分成训练集和测试集，而 REFUGE 以及 DRISHTI-GS1 作为测试集. 不同数 据集中眼底图像如图 11 所示.

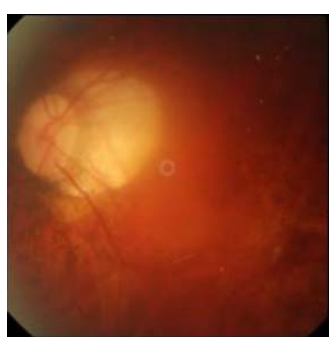

a. LAG database 数据集

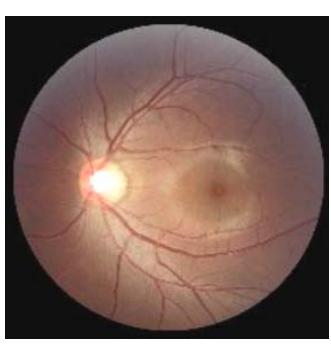

c. REFUGE 测试集

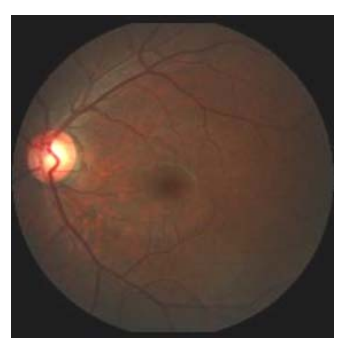

b. REFUGE 训练集

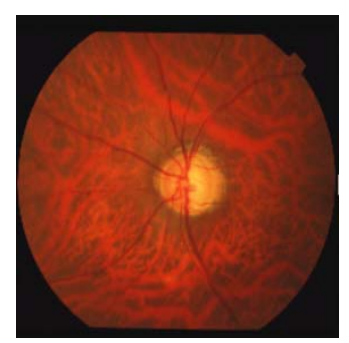

d. DRISHTI-GS1 数据集
图 11 不同眼底数据集眼底图像

\section{2 实验参数设置}

本文实验都是基于 Python 语言以及 PyTorch 深度学习框架实现, 并采用 RTX 2080Ti GPU 完成 加速训练.

在模型训练阶段，随机对比度、随机亮度、随 机 gamma 变换、随机饱和度、随机裁剪、随机旋 转和水平翻转这些数据增强方法被用于增强训练
样本多样性. 训练分类网络所采用的优化器为 SGD, 训练批量大小为 64 , 输人图像的像素大小 为 $224 \times 224 \times 3$, 训练总轮数为 200 , 初始学习率为 0.001 , 学习率在第 100 和第 150 轮依次减小 $90 \%$.

为了加速模型收敛并缓解数据规模偏小的问 题, 本文使用迁移学习来初始化模型参数. 模型 参数中卷积层部分由基于 ImageNet 数据集训练 好的分类模型迁移而来, 而全连接层参数采用 Kaiming 初始化. 在初始训练阶段, 本文方法冻 结住卷积层部分参数, 而只更新全连接层参数; 当模型训练收玫后, 对模型中后层参数解冻, 解 冻参数均参与更新.

\section{3 实验结果}

分类模型的主要评价指标为准确率, 而对于 青光眼分类任务, 非患病(负类)图像占据很少一部 分, 当预测偏向非患病类别所得准确率也可较高. 因此, 本文主要通过敏感性 $S$ 、特异性 $P$ 以及 AUC 指标评估不同青光眼篮查方法的性能差异, 敏感 性和特异性的数学表达式分别为

$$
\begin{aligned}
& S=\frac{\mathrm{TP}}{\mathrm{TP}+\mathrm{FN}} \\
& P=\frac{\mathrm{TN}}{\mathrm{TN}+\mathrm{FP}}
\end{aligned}
$$

其中, TP, TN, FP, FN 分别表示真正例(true positive, TP)、真反例(true negative, TN)、假正例(false positive, FP)和假反例(false negative, FN).

\subsection{1 分类性能评估}

基于不同数据集分别对不同青光眼耖查方法 在敏感性、特异性以及 AUC 指标上进行对比, 结 果分别如表 1 表 3 所示.

由表 1 表 3 可知, 针对 LAG database 眼底数 据集 ${ }^{[32]}$, 本文方法在各项指标上都达到最优. 该 数据集中对比方法均为基于卷积神经网络的深度 学习模型. Chen 等 ${ }^{[35]}$ 和 $\mathrm{Li}$ 等 ${ }^{[36]}$ 方法分别为 6 层和 22 层卷积网络, 可以发现前者各项指标均偏低, 这可能是由于模型深度过浅导致模型表征能力欠 佳. AG-CNN 模型 ${ }^{[32]}$ 得出结果仅次于本文方法, 它

\begin{tabular}{|c|c|c|c|}
\hline 方法 & $S$ & $P$ & AUC \\
\hline $\mathrm{AG}-\mathrm{CNN}^{[32]}$ & $\underline{0.954}$ & $\underline{0.952}$ & $\underline{0.975}$ \\
\hline Chen 等 ${ }^{[35]}$ & 0.906 & 0.882 & 0.956 \\
\hline $\mathrm{Li}$ 等 ${ }^{[36]}$ & 0.914 & 0.884 & 0.960 \\
\hline 本文 & 0.970 & 0.983 & 0.996 \\
\hline
\end{tabular}

表 1 不同方法在 LAG database 数据集对比结果

注. 加粗表示单项指标最高结果, 下划线为次优结果. 
表 2 不同方法在 DRISHTI-GS1 数据集对比结果

\begin{tabular}{lccc}
\hline \multicolumn{1}{c}{ 方法 } & $S$ & $P$ & AUC \\
\hline DeepCDR $^{[13]}$ & $\mathbf{0 . 9 4 7}$ & 0.536 & 0.848 \\
WaveLet $^{[20]}$ & 0.816 & $\underline{0.615}$ & 0.767 \\
Gabor $^{[37]}$ & $\underline{0.921}$ & $\underline{0.615}$ & $\underline{0.877}$ \\
本文 & 0.809 & $\mathbf{0 . 8 4 6}$ & $\mathbf{0 . 8 8 3}$ \\
\hline
\end{tabular}

注. 加粗表示单项指标最高结果, 下划线为次优结果.

表 3 不同方法在 REFUGE 数据集对比结果

\begin{tabular}{lccc}
\hline \multicolumn{1}{c}{ 方法 } & $S$ & $P$ & AUC \\
\hline CUHKMED $^{[33]}$ & $\underline{0.843}$ & - & 0.924 \\
VRT $^{[33]}$ & $\mathbf{0 . 9 5 0}$ & - & $\mathbf{0 . 9 7 2}$ \\
CVBLab $^{[33]}$ & 0.832 & - & 0.939 \\
本文 & $\mathbf{0 . 9 5 0}$ & $\mathbf{0 . 8 9 4}$ & $\underline{0.956}$ \\
\hline
\end{tabular}

注. 加粗表示单项指标最高结果, 下划线为次优结果.

首先通过全卷积网络预测出注意力区域，然后通 过该区域对分类网络特征图加权，这在一定程度 保留了特征图上分类相关特征区域并抑制非相关 区域，从而提升了整体分类性能.

本文方法不同于 $\mathrm{AG}-\mathrm{CNN}$ 那样通过单独分支 预测注意力区域，也不同于常规卷积模型 ${ }^{[35-36]}$, 而 是通过语义特征图结合浅层特征图生成注意力图, 自动优化浅层特征，增强分类相关特征中细节区 域. 这样的设计使得最终的 AUC 和敏感性指标比 AG-CNN 提升接近 2\%, 而特异性提升接近 $3 \%$.

不同篮查方法在 DRISHTI-GS1 数据集 ${ }^{[34]}$ 上的 对比结果如表 2 所示. 结果显示, DeepCDR 方法 ${ }^{[13]}$ 获得最高的敏感性, Gabor 方法 ${ }^{[37]}$ 次之，而本文方 法在特异性及 AUC 上统计结果最好, 这表明 DeepCDR 等其他模型具有一定的预测偏见，偏向 于多数的负类. 此外, DRISHTI-GS1 数据集测试数 据与模型训练数据成像差异较大, 较大的数据域 差异对模型预测鲁棒性有一定的影响。

不同䇥查方法在 REFUGE 数据集 ${ }^{[33]}$ 对比结果 如表 3 所示. 可以看出, 本文方法在敏感性以及 AUC 指标上达到最优和次优的性能. VRT 团队 ${ }^{[33]}$ 借助丰富的私有青光眼篮查数据以及伪标签技术, 使得模型在验证集与测试集上均达到最高水平，这 也表明数据的多样性以及数据量对模型性能上限有 较大的影响. CVBLab ${ }^{[33]}$ 采用的是多模型融合策略, 所融合的模型包含 VGG19, ResNet-50, Inception v3 以及 Xception 这些经典分类模型. 本文篮查模型 为单模型，在敏感性指标上远高于 CVBLab，在 AUC 上高出 $1.7 \%$. CUHKMED 等 ${ }^{[33]}$ 提出一种对抗
视盘视杯分割模型, 并仅使用杯盘比指标作为青 光眼患病可能性，在验证集上 AUC 指标显得偏低. 2.3.2 决策区域可视化

本文对篮查模型中第 3 组及第 4 组特征分别应 用注意力机制, 所得注意力图为单通道激活图, 注 意力图激活范围为 $0 \sim 1,0$ 表示无任何激活, 1 表示 对应区域与青光眼检测高度相关. 为方便可视化 注意力图对应眼底图像激活区域, 首先采用双线 性插值技术缩放注意力图至眼底图像同等大小, 然后通过伪彩色处理注意力图得出彩色热图, 最 后按一定比例融合眼底图像以及注意力热图, 得 出检测模型决策区域可视化结果，其中高亮区域 对应模型重点关注区域. 通过基于 Grad-CAM 方 法 $^{[38]}$ 与本文方法生成的模型决策区域可视化结果 对比如图 12 所示, 其中数据由上向下依次来自 REFUGE, DRISHTI-GS1 和 LAG database 数据集.

从实验结果可以发现，基于 Grad-CAM 方法 得出的决策区域与模型注意力图激活区域较为吻 合, 2 种方法激活区域均集中于视盘上. 但与 GradCAM 覆盖整个视盘相比, 本文注意力图得出的激 活区域更为精细, 且不同注意力激活热图具有一 定互补性.

此外, 从图 12 中眼底图像决策区域可视化可 以发现, 第 1 组注意力模块关注区域偏向于视盘上 下两侧且集中于盘沿区域，这与临床诊断时关注 盘沿区域是否出现盘沿分布病变、视盘出血或视盘 旁萎缩来诊断患病风险较为一致. 而第 2 组注意力 模块更关注视盘整体或中间视杯区域，这与临床 诊断时检查视杯是否出现扩张趋势或视神经纤维 层萎缩较为相似. 这表明模型自我学习出的关注 区域也与临床诊断重点关注区域非常吻合, 而通 过可视化分析模型决策激活区域可有助于临床医 生从图像中发掘出更多未被发现的诊断判别区域, 进而得出类似杯盘比等关键临床指标, 以辅助篮 查, 这将对医生深人理解青光眼眼组织形态改变 发挥积极作用. 由此可见, 语义特征图引导的注意 力机制不仅对最终的青光眼篮查有一定提升作用, 也给出了更为精细的决策区域可视化结果, 可为 青光眼的临床辅助篮查提供有力的帮助.

\subsection{3 临床数据测试}

为进一步验证本文青光眼篮查模型的有效性, 基于从中南大学湘雅二医院收集的 825 幅眼底图 像数据进行测试实验, 数据包含 311 幅疑似青光 眼和 514 幅非青光眼眼底图像. 测试结果显示, 


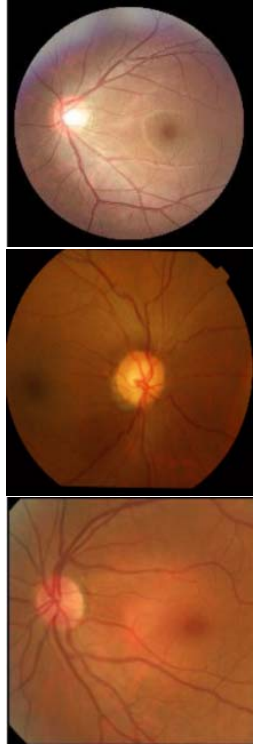

a. 输人眼底图像

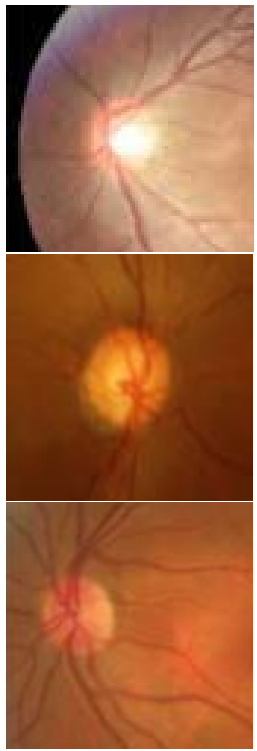

b. 感兴趣区域
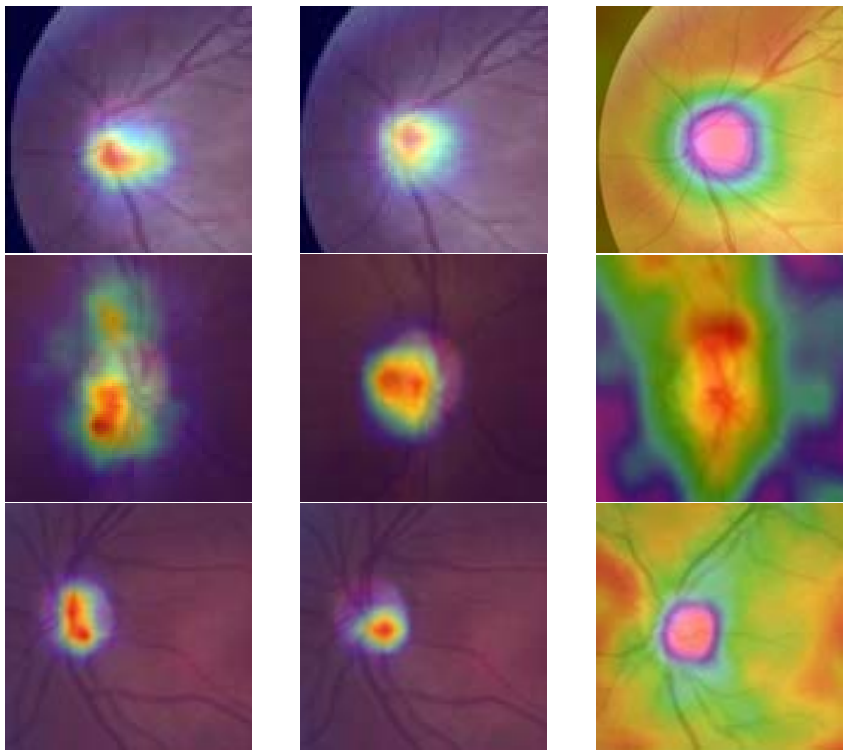

c. 注意力模块 1 激活图 d. 注意力模块 2 激活图 e. Grad-CAM 类激活图

图 12 模型决策区域可视化

AUC、敏感性和特异性指标分别为 $0.83,0.75$ 和 0.87. 从可视化模型决策激活区域可发现模型仍依 靠视盘周边区域病变做出决策，这也与临床诊断 关注区域较为一致，部分测试数据及模型注意力 热图激活区域可视化结果如图 13 所示.

\section{4 讨 论}

为了验证本文注意力机制模块的有效性，基

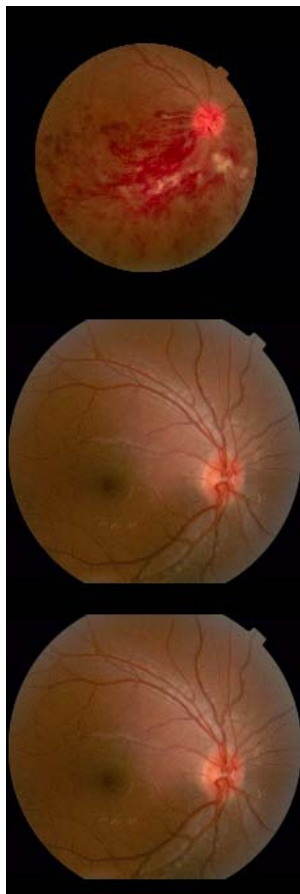

a. 输人眼底图像

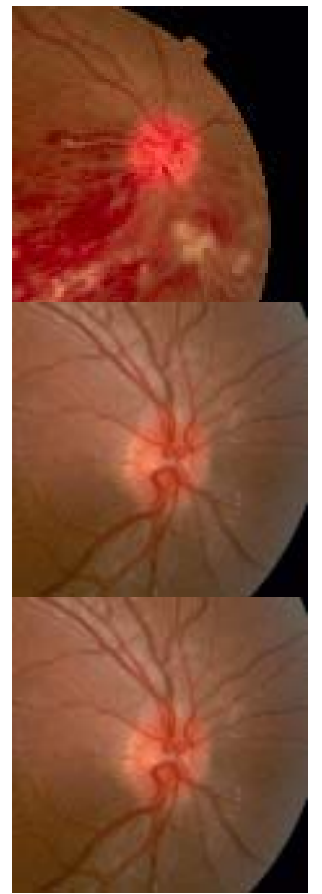

b. 感兴趣区域
于 LAG database 数据集, 以原始 VGG16 网络为基 准, 分别采用其第 2 和第 3 层特征以及第 2 和第 4 层特征组合构造注意力机制模块, 并且与本文方 法进行了对比, 实验结果的敏感性、特异性以及 AUC 指标如表 4 所示. 从实验结果可以看出, 无论 采用哪 2 层特征组合构造注意力机制模块, 其实验 结果都较原始 VGG16 网络有明显提升, 且本文方

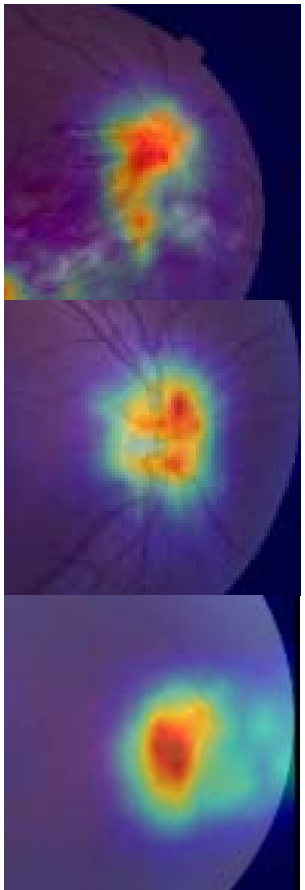

c. 注意力模块 1 激活图

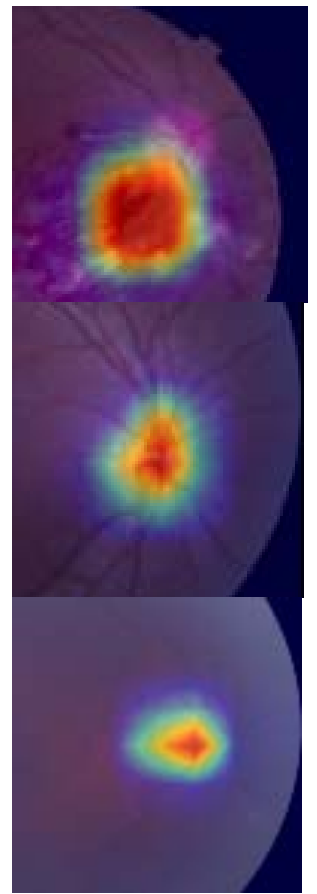

d. 注意力模块 2 激活图

图 13 临床测试数据及模型决策区域可视化结果 
表 4 不同特征组合构造注意力机制模块的对比结果

\begin{tabular}{lccc}
\hline \multicolumn{1}{c}{ 方法 } & $S$ & $P$ & AUC \\
\hline VGG16 $^{[27]}$ & 0.953 & $\underline{0.976}$ & 0.994 \\
VGG16+第 2, 3 层特征加 & 0.962 & 0.971 & $\underline{0.995}$ \\
人注意力机制 & & & \\
VGG16+第 2, 4 层特征加 & $\underline{0.965}$ & 0.944 & 0.993 \\
人注意力机制 & $\mathbf{0 . 9 7 0}$ & $\mathbf{0 . 9 8 3}$ & $\mathbf{0 . 9 9 6}$ \\
本文 &
\end{tabular}

注. 加粗表示单项指标最高结果, 下划线为次优结果.

法在各项指标上都达到最佳，也佐证了采用第 3 和 第 4 层特征构造注意力机制模块可以兼顾一定的 语义性与尺度大小，并可得到最佳效果的分析.

此外，设计分割定位模型的目的是获取以视 盘为中心的感兴趣区域，并将其作为分类网络的 输人. 因为青光眼病变主要发生在视杯和视盘区 域，如果直接以全局眼底图像作为分类网络的输 人，可能造成大量无意义的计算消耗，影响对青光 眼的篮查性能. 而将全局眼底图像下采样作为网 络输人尺寸 $(224 \times 224 \times 3)$ 会造成像素损失，这也 在一定程度上降低了分类性能. 基于 LAG database 数据集分割定位模块的消融实验结果如表 5 所示. 如果移除分割定位模块, 会导致实验结果在 敏感性、特异性以及 AUC 上分别下降 2.99\%, $3.97 \%$ 和 $0.60 \%$. 这也佐证了分割定位模块对于语 义特征图引导的青光眼篎查方法的贡献, 进一步 说明了青光眼的关键篎查证据主要集中在视盘区 域附近，将聚焦于视盘区域的感兴趣区域作为分 类网络的输人, 可以取得更优的青光眼篮查效果.

表 5 基于 LAG database 数据集的 分割定位模块消融实验结果

\begin{tabular}{lccc}
\hline \multicolumn{1}{c}{ 方法 } & $S$ & $P$ & AUC \\
\hline 移除分割定位模块 & 0.941 & 0.944 & 0.990 \\
本文 & $\mathbf{0 . 9 7 0}$ & $\mathbf{0 . 9 8 3}$ & $\mathbf{0 . 9 9 6}$ \\
\hline
\end{tabular}

注. 加粗表示单项指标最高结果.

基于 LAG database, REFUGE 数据集的实验结 果均表明，语义特征图引导的青光眼篮查方法可 以在敏感性、特异性和 AUC 3 个指标上取得总体 最佳的效果, 并且优于以 AG-CNN 为代表的其他 算法. 当用于测试 DRISHTI-GS1 数据集和中南大 学湘雅二医院收集眼底图像数据时, 各项指标比 LAG-database 数据集有一定下滑. 经分析可能造 成精度下降的主要原因为所收集的影像数据成像 与模型训练数据相差较大, 从而对模型造成较大 干扰，这也影响了模型测试鲁棒性. 因此，进一步
完善训练数据覆盖面、研究统一数据形态的数据变 换方法或者提升模型的可迁移性, 对模型真正落 地十分重要, 并且成为下一步的工作内容. 此外, 已有青光眼篎查方法未能有效地结合光学相干断 层扫描(optical coherence tomography, OCT)或者临 床病史等数据, 综合研究多模态数据对青光眼笁 查准确性与鲁棒性有重要意义.

\section{3 结 语}

本文针对已有卷积分类方法未能着重关注细 微病变相关区域导致模型易过拟合且模型决策解 释性区域不明确的问题, 提出了一种语义特征图 引导的青光眼篮查模型. 语义特征图引导的注意 力模块融合了语义判别特征与浅层细节特征, 以 生成注意力图强化浅层特征分类相关区域, 并抑 制非相关区域, 以增强模型预测准确性. 通过可视 化注意力激活热图可较好地得出模型决策证据图 谱. 相比已有可视化方法, 本文方法得出的模型决 策区域更为精细，且符合临床诊断关注区域. 此 外, 本文还提出利用 DeepLab v3+模型对视盘区域 进行分割, 将视盘定位转换为视盘分割问题求解; 采用 MobileNet 作为分割网络特征提取主干模块, 使得整体分割模型轻量化. 在多个数据集上的实 验结果证实, 相比已有方法, 本文方法具有相对较 优的性能, 为临床青光眼的自动篮查工作奠定了 良好基础.

\section{参考文献(References):}

[1] Tham Y C, Li X, Wong T Y, et al. Global prevalence of glaucoma and projections of glaucoma burden through 2040: a systematic review and meta-analysis[J]. Ophthalmology, 2014, 121(11): 2081-2090

[2] Shen S Y, Wong T Y, Foster P J, et al. The prevalence and types of glaucoma in Malay people: the Singapore Malay eye study[J]. Investigative Ophthalmology \& Visual Science, 2008, 49(9): 3846-3851

[3] Jonas J B, Budde W M, Panda-Jonas S. Ophthalmoscopic evaluation of the optic nerve head[J]. Survey of Ophthalmology, 1999, 43(4): 293-320

[4] Zhu X L, Rangayyan R M, Ells A L. Detection of the optic nerve head in fundus images of the retina using the hough transform for circles[J]. Journal of Digital Imaging, 2010, 23(3): 332-341

[5] Jonas J B, Bergua A, Schmitz-Valckenberg P, et al. Ranking of optic disc variables for detection of glaucomatous optic nerve damage[J]. Investigative Ophthalmology \& Visual Science, 
2000, 41(7): 1764-1773

[6] Walter T, Klein J C. Segmentation of color fundus images of the human retina: Detection of the optic disc and the vascular tree using morphological techniques[C] //Proceedings of International Symposium on Medical Data Analysis. Heidelberg: Springer, 2001: 282-287

[7] Nayak J, Acharya U R, Bhat P S, et al. Automated diagnosis of glaucoma using digital fundus images[J]. Journal of Medical Systems, 2008, 33(5): 337-346

[8] $\mathrm{Xu} \mathrm{J,} \mathrm{Chutatape} \mathrm{O,} \mathrm{Sung} \mathrm{E,} \mathrm{et} \mathrm{al.} \mathrm{Optic} \mathrm{disk} \mathrm{feature} \mathrm{extraction}$ via modified deformable model technique for glaucoma analysis[J]. Pattern Recognition, 2007, 40(7): 2063-2076

[9] Li H Q, Chutatape O. Boundary detection of optic disk by a modified ASM method[J]. Pattern Recognition, 2003, 36(9): 2093-2104

[10] Kim S K, Kong H J, Seo J M, et al. Segmentation of optic nerve head using warping and RANSAC[C] //Proceedings of Annual International Conference of the IEEE Engineering in Medicine and Biology Society. Los Alamitos: IEEE Computer Society Press, 2007: 900-903

[11] Cheng J, Liu J, Xu Y W, et al. Superpixel classification based optic disc and optic cup segmentation for glaucoma screening[J]. IEEE Transactions on Medical Imaging, 2013, 32(6): 1019-1032

[12] Zheng Y J, Stambolian D, O’Brien J, et al. Optic disc and cup segmentation from color fundus photograph using graph cut with priors[C] //Proceedings of International Conference on Medical Image Computing and Computer-Assisted Intervention. Heidelberg: Springer, 2013: 75-82

[13] Fu H Z, Cheng J, Xu Y W, et al. Joint optic disc and cup segmentation based on multi-label deep network and polar transformation[J]. IEEE Transactions on Medical Imaging, 2018, 37(7): 1597-1605

[14] Sevastopolsky A. Optic disc and cup segmentation methods for glaucoma detection with modification of U-Net convolutional neural network $[\mathrm{J}]$. Pattern Recognition and Image Analysis, 2017, 27(3): 618-624

[15] Singh V K, Rashwan H A, Saleh A, et al. REFUGE Challenge 2018-Task 2: deep optic disc and cup segmentation in fundus images using U-net and multi-scale feature matching networks[OL]. [2020-07-04]. https://arxiv.org/abs/1807.11433

[16] Xu Y W, Lin S, Wong D W K, et al. Efficient reconstruction-based optic cup localization for glaucoma screening[C] // Proceedings of International Conference on Medical Image Computing and Computer-Assisted Intervention. Heidelberg: Springer, 2013: 445-452

[17] Agarwal A, Gulia S, Chaudhary S, et al. A novel approach to detect glaucoma in retinal fundus images using cup-disk and rim-disk ratio[C] //Proceedings of the 4th International Work Conference on Bioinspired Intelligence. Los Alamitos: IEEE Computer Society Press, 2015: 139-144

[18] Dua S, Acharya U R, Chowriappa P, et al. Wavelet-based energy features for glaucomatous image classification[J]. IEEE Transactions on Information Technology in Biomedicine, 2012, 16(1): $80-87$

[19] Gayathri Devi T M, Sudha S, Suraj P. Artificial neural networks in retinal image analysis[C] //Proceedings of the 3rd International Conference on Signal Processing, Communication and Networking. Los Alamitos: IEEE Computer Society Press, 2015: 1-6

[20] Koh J E W, Acharya U R, Hagiwara Y, et al. Diagnosis of retinal health in digital fundus images using continuous wavelet transform (CWT) and entropies[J]. Computers in Biology and Medicine, 2017, 84: 89-97

[21] Noronha K P, Acharya U R, Nayak K P, et al. Automated classification of glaucoma stages using higher order cumulant features[J]. Biomedical Signal Processing and Control, 2014, 10: 174-183

[22] Mookiah M R K, Acharya U R, Lim C M, et al. Data mining technique for automated diagnosis of glaucoma using higher order spectra and wavelet energy features $[\mathrm{J}]$. Knowledge-Based Systems, 2012, 33: 73-82

[23] Fu H Z, Cheng J, Xu Y W, et al. Disc-aware ensemble network for glaucoma screening from fundus image[J]. IEEE Transactions on Medical Imaging, 2018, 37(11): 2493-2501

[24] Chai Y D, Liu H Y, Xu J. Glaucoma diagnosis based on both hidden features and domain knowledge through deep learning models[J]. Knowledge-Based Systems, 2018, 161: 147-156

[25] Chen L C, Papandreou G, Kokkinos I, et al. DeepLab: semantic image segmentation with deep convolutional nets, atrous convolution, and fully connected CRFs[J]. IEEE Transactions on Pattern Analysis and Machine Intelligence, 2018, 40(4): 834-848

[26] Howard A G, Zhu M L, Chen B, et al. MobileNets: efficient convolutional neural networks for mobile vision applications[OL]. [2020-07-04]. https://arxiv.org/abs/1704.04861

[27] Simonyan K, Zisserman A. Very deep convolutional networks for large-scale image recognition[OL]. [2020-07-04]. https:// arxiv.org/abs/1409.1556

[28] He K M, Zhang X Y, Ren S Q, et al. Deep residual learning for image recognition[C] //Proceedings of the IEEE Conference on Computer Vision and Pattern Recognition. Los Alamitos: IEEE Computer Society Press, 2016: 770-778

[29] Huang G, Liu Z, van der Maaten L, et al. Densely connected convolutional networks[C] //Proceedings of the IEEE Conference on Computer Vision and Pattern Recognition. Los Alamitos: IEEE Computer Society Press, 2017: 2261-2269

[30] Chollet F. Xception: deep learning with depthwise separable convolutions[C] //Proceedings of the IEEE Conference on Computer Vision and Pattern Recognition. Los Alamitos: IEEE Computer Society Press, 2017: 1800-1807

[31] Long J, Shelhamer E, Darrell T. Fully convolutional networks for semantic segmentation[J]. IEEE Transactions on Pattern Analysis and Machine Intelligence, 2017, 39(4): 640-651

[32] Li L, Xu M, Wang X F, et al. Attention based glaucoma detection: a large-scale database and $\mathrm{CNN}$ model[C] //Proceedings of the IEEE/CVF Conference on Computer Vision and Pattern Recognition. Los Alamitos: IEEE Computer Society Press, 2019: 10563-10572

[33] Orlando J I, Fu H Z, Breda J B, et al. REFUGE challenge: a unified framework for evaluating automated methods for glaucoma assessment from fundus photographs[J]. Medical Image 
Analysis, 2020, 59: 101570

[34] Sivaswamy J, Krishnadas S R, Chakravarty A, et al. A comprehensive retinal image dataset for the assessment of glaucoma from the optic nerve head analysis[J]. JSM Biomedical Imaging Data Papers, 2015, 2(1): 1004

[35] Chen X Y, Xu Y W, Yan S C, et al. Automatic feature learning for glaucoma detection based on deep learning[C] //Proceedings of the International Conference on Medical Image Computing and Computer-Assisted Intervention. Heidelberg: Springer, 2015: 669-677

[36] Li Z X, He Y F, Keel S, et al. Efficacy of a deep learning sys- tem for detecting glaucomatous optic neuropathy based on color fundus photographs[J]. Ophthalmology, 2018, 125(8): 1199-1206

[37] Acharya U R, Ng E Y K, Eugene L W J, et al. Decision support system for the glaucoma using Gabor transformation[J]. Biomedical Signal Processing and Control, 2015, 15: 18-26

[38] Selvaraju R R, Cogswell M, Das A, et al. Grad-CAM: visual explanations from deep networks via gradient-based localization[C] //Proceedings of the IEEE International Conference on Computer Vision. Los Alamitos: IEEE Computer Society Press, 2017, 1: 618-626 